1. Allenmark, S. and Hagberg, C.-E. Acta Chem. Scand. 22 (1968) 1461.

2. Mislow, K., Simmons, T., Melillo, J. T. and Ternay, A. L. J. Am. Chem. Soc. 86 (1964) 1452.

3. Mislow, K. Record Chem. Progr. 28 (1967) 229.

4. Landini, D., Montanari, F., Modena, G. and Scorrano, G. Chem. Commun. 196886.

5. Allenmark, S. Arkiv Kemi 26 (1967) 37.

6. Allenmark, S. and Johnsson, H. Acta Chem. Scand. 21 (1967) 1672.

7. Long, F. A. and MeIntyre, D. J. Am. Chem. Soc. 76 (1954) 3243.

8. Paul, M. A. and Long, F. A. Chem. Rev. 57 (1957) 17.

Received May 16, 1968.

\section{An Alternate Route for the Formation of Malonate in Penicillium islandicum}

\section{STEN GATENBECK and ANN KERSTI MAHLÉN}

Division of Biochemistry, Chemical Centre, Box 740, S.220 07 Lund 7, Sweden

$\mathbf{T}$ he main pathway for the formation of malonyl coenzyme $A$ in most organisms, is the biotin dependent addition of $\mathrm{CO}_{2}$ to acetyl coenzyme A. ${ }^{1}$ Related to this reaction is the transcarboxylation between acetyl coenzyme $A$ and methylmalonyl coenzyme A which has been demonstrated with an enzyme preparation from wheat germ. ${ }^{2}$

Free malonate is a common metabolite in higher plants. The occurrence of malonate can be looked upon as the result of the action of thiolesterase on malonyl coenzyme A. The presence of malonyl coenzyme A thiolesterase has been shown in several investigations.

Recently de Vellis et al. ${ }^{3}$ have studied the malonic acid synthesis in the bush bean (Phaseolus vulgaris). With crude preparation from the roots using a variety of labelled substrates they came to the conclusion that the malonate was formed from oxalacetate by $\alpha$-decarboxylation. Shannon et $a l .{ }^{4}$ have partially purified the enzyme from bush bean roots and they have been able to show that the enzyme is of the peroxidase type requiring the presence of manganous ions and hydrogen peroxide.

Many microorganisms especially among fungi produce in addition to fatty acids large amounts of other substances of acetate-polymalonate origin. The origin of the malonate itself in these substances has not been investigated, possibly due to the general opinion that it is formed by carboxylation of acetyl coenzyme A. In the formation of the acetate-polymalonate products as well as in fatty acid synthesis the reacting malonyl coenzyme A units have undergone decarboxylations and consequently the final products do not represent the intact malonate structure.

Free malonate is a rare product among microorganisms. Penicillium islandicum, however, produces when grown on CzapekDox medium an acid polysaccharide, islandic acid, ${ }^{5}$ composed of glucose and malonic acid in the approximate molecular ratio 1:1. In addition to islandic acid $P$. islandicum produces considerable amounts of anthraquinone derivatives derived from acetatepolymalonate. ${ }^{6}$

In the following we have studied the possible conversion of oxalacetate to malonate. As oxalacetate is a labile compound and penetrates through the cell wall very poorly, uniformly C-14 labelled aspartate and 2,3-C-14 labelled fumarate have been used as tracer substrates. Theoretically, four possible metabolic routes can be set up from oxalacetate to malonyl coenzyme $\mathbf{A}$, the latter compound being the likely reacting metabolite in the esterification of the hydroxyl groups of the polysaccharide. The four possible pathways are: 1) Oxidative decarboxylation, analogous to the thiamine pyrophosphatelipoic acid system in the oxidative decarboxylation of pyruvate and $\alpha$-ketoglutarate, i.e. an immediate formation of malonyl coenzyme A. 2) Peroxidase type of oxidative decarboxylation of oxalacetate to malonate followed by the action of thiokinase. 3) Reduction of oxalacetate to malate prior to cleavage to glyoxalate and acetyl coenzyme $A$, the latter being carboxylated to malonyl coenzyme A. 4) $\beta$ Decarboxylation of oxalacetate to pyruvate which is subsequently oxidatively decarboxylated to acetyl coenzyme A 


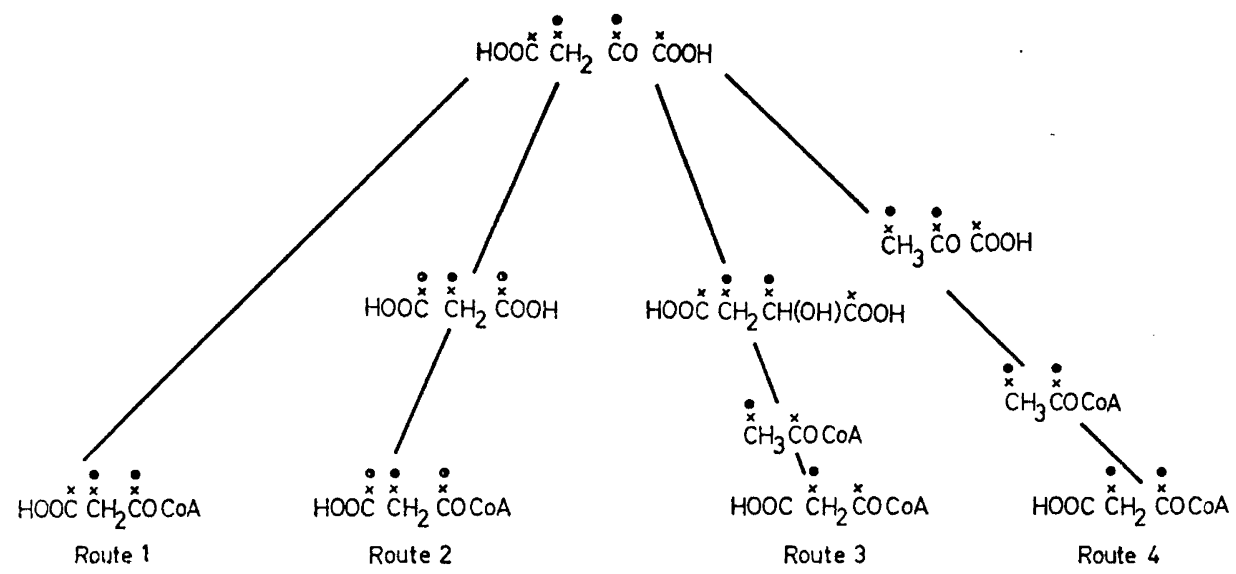

Fig. 1. Possible pathways of malonyl coenzyme A formation from oxalacetate.

followed by carboxylation to malonyl coenzyme A.

From Fig. 1 is seen that by using uniformly labelled aspartate as substrate the labelling will appear uniformly distributed in the malonate structure only if the malonate is formed by an $\alpha$-decarboxylation of oxalacetate. However, the labelling pattern will not distinguish between route 1 and 2 . When using fumarate$2,3{ }^{14} \mathrm{C}$ as substrate the labelling pattern in the formed malonate will be identical for routes 1,2 , and 4 because of randomization of the radioactivity in the carboxyl groups of malonic acid after hydrolysis. Route 3 will in this case only give rise to ${ }^{14} \mathrm{C}$ in the methylene group of malonate.
In the experiments the labelled substrates were added to growing cultures producing islandic acid. After one day of incubation the islandic acid was isolated and hydrolyzed to malonic acid and glucose. The radioactivities of the malonate and its individual carbon atoms were determined (Table 1).

The ${ }^{14} \mathrm{C}$ content of the methylene group of malonate is very close to twice that of each carboxyl group when fumarate-2,3-14 $\mathrm{C}$ is used as precursor. This indicates that route 3 does not contribute to the formation of malonate. The ${ }^{14} \mathrm{C}$ distribution in malonate from uniformly labelled aspartate was determined in two experiments. In one of them each carboxyl group was carrying

Table 1. Distribution of ${ }^{14} \mathrm{C}$ in malonate after incubation with $\mathrm{L}_{4}$-aspartate-U $-{ }^{14} \mathrm{C}$ and fumarate$2,3{ }^{14} \mathrm{C}$.

\begin{tabular}{|c|c|c|c|}
\hline${ }^{14} \mathrm{C}$ precursor & $\begin{array}{l}\text { Malonate } \\
\text { cpm/mmole }\end{array}$ & $\begin{array}{l}\text { Carboxyl group } \\
\text { cpm/mmole }\end{array}$ & $\begin{array}{l}\text { Methylene group } \\
\text { cpm/mmole }\end{array}$ \\
\hline $\mathrm{I}_{4}$-Aspartate-U-14C & $34.6 \times 10^{3}$ & $11.5 \times 10^{3}$ & $11.6 \times 10^{3 a}$ \\
\hline L-Aspartate-U.-14 C & $8.95 \times 10^{3}$ & $2.7 \times 10^{3}$ & $3.3 \times 10^{3}$ \\
\hline Fumarate-2,3 ${ }^{14} \mathrm{C}$ & $54.0 \times 10^{4}$ & $13.1 \times 10^{4}$ & $27.9 \times 10^{4 a}$ \\
\hline
\end{tabular}

\footnotetext{
${ }^{a}$ Calculated as the difference between the radioactivities of the malonate and the carboxyl groups.
}

Acta Chem. Scand. 22 (1968) No. 5 
exactly a third of the total radioactivity of the malonate which is only compatible with the formation of malonate exclusively by oxidative $\alpha$-decarboxylation, i.e according to route 1 or 2 . In the second experiment using uniformly labelled aspartate a slightly higher radioactivity was obtained in the methylene group as compared to the radioactivity of each carboxyl group which might indicate some contribution of route 4 to the malonate formation. Calculations from the figures in the table show that $77 \%$ of the labelling in the malonate is derived from route 1 or 2 and $23 \%$ from route 4.

The experiments demonstrate thus that there is a metabolic pathway in $P$. islandi. cum from oxalacetate to malonate in which oxidative $\alpha$-decarboxylation of oxalacetate is involved. The question whether this reaction is related to the oxidative decarboxylation of pyruvate or is of peroxidase character can only be settled when the reaction is studied in a cell free system.

1. Gibson, D. M., Titchener, E. B. and Wakil, S. J. Biochem. Biophys. Acta 30 (1958) 376.

2. Hatch, M. D. and Stumpf, P. K. J. Biol. Chem. 236 (1961) 2879.

3. de Vellis, J., Shannon, L. M. and Lew, J. Y. Plant Physiol. 38 (1963) 686.

4. Shannon, L. M., de Vellis, J. and Lew, J. Y. Plant Physiol. 38 (1963) 691.

5. Ebert, E. and Zenk, M. H. Phytochemistry. 6 (1967) 309.

6. Gatenbeck, S. Acta Chem. Scand. 16 (1962) 1053.

7. Van Slyke, D. D. and Folch, J. J. Biol. Chem. 136 (1940) 509.

8. Phares, E. F. Arch. Biochem. Biophys. 33 (1951) 173.

Received May 28, 1968.

\section{Chemical Studies on Lichens}

\section{2.* A New Lichen Xanthone from Lecanora reuteri JOHAN SANTESSON}

The Institute of Chemistry, University of Uppsala, Box 531, S-751 21 Uppsala, Sweden

A rapidly growing number of xanthones, most of which chlorinated, are known from lichens. Some examples are lichexanthone (II), 1 thiophanic acid (III), ${ }^{2-4}$ arthothelin (IV), ${ }^{5-7}$ and thuringion (V). ${ }^{7-8}$ All these are derived from the same parent compound norlichexanthone (I, 1,3,6-trihydroxy-8-methylxanthene-9H-9-one).

During a screening of Lecanora species by "lichen mass spectrometry" it was found that the spectrum of $L$. reuteri Schaer. (Fig. 1) exhibited a strong peak at $m / e 258$ which suggested the presence of norlichexanthone $(\mathrm{M}=258)$. A peak at $m / e$ 360 with satellite peaks at $m / e 362,364$, and 366 indicated the presence of the corresponding trichlorinated xanthone arthothelin (IV).<smiles>[R]Oc1cc(C)c2c(=O)c3c(O)cc([R20])cc3oc2c1</smiles><smiles></smiles>

* Part 11. Arkiv Kemi. In press. 